\title{
ON WEIGHTED NORM INEQUALITIES FOR \\ THE HILBERT TRANSFORM \\ OF FUNCTIONS WITH MOMENTS ZERO
}

BY

ERNST ADAMS

ABSTRACT. Let $\tilde{f}$ denote the Hilbert transform of $f$, i.e.

$$
\tilde{f}(x)=\text { p.v. } \int \frac{f(t)}{x-t} d t
$$

and let $1<p<\infty$. A weight function $w$ is shown to satisfy

$$
\int|\tilde{f}(x)|^{p} w(x) d x \leqslant C \int|f(x)|^{p} w(x) d x
$$

for all $f$ with the first $N$ moments zero, if and only if it is of the form $w(x)=$ $|q(x)|^{p} U(x)$, where $q$ is a polynomial of degree at most $N$ and $U \in A_{p}$.

1. The problem is to determine for given $p, 1<p<\infty$, all weights $w$, which satisfy

$$
\int|\tilde{f}(x)|^{p} w(x) d x \leqslant C \int|f(x)|^{p} w(x) d x
$$

for some positive constant $C$ and for all functions $f$ with $\int|f(x)|(1+|x|)^{N} d x<\infty$ and for which $\int x^{k} f(x) d x=0,0 \leqslant k \leqslant N$, for a nonnegative integer $N$.

The case where no restrictions on the moments of $f$ are imposed and $p=2$ was solved by Helson and Szegö in 1960 [5], and for general $p, 1<p<\infty$, by Hunt, Muckenhoupt and Wheeden in 1973 [6]. In that case $w$ satisfies (1.1) if and only if $w \in A_{p}$, i.e. if there exists a constant $C$ such that for all intervals $I$

$$
\frac{1}{|I|} \int_{I} w(x) d x\left(\frac{1}{|I|} \int_{I} w(x)^{-1 /(p-1)} d x\right)^{p-1} \leqslant C .
$$

Of course one expects more admissible weights if there are fewer functions $f$ allowed in (1.1). Indeed it is easy to see, and we will show it in a moment, that weights of the form $w(x)=|q(x)|^{p} U(x)$ satisfy (1.1), where $q$ is a polynomial with complex coefficients of degree $N+1$ and $U \in A_{p}$, when $f$ has all up to the $N$ th moments zero.

As it turns out, these are in fact all the weights. This was shown for $p=2$ in 1967 by Helson and Sarason [4], see also Arocena, Cotlar and Sadosky [1]. They used

Received by the editors October 28, 1980.

1980 Mathematics Subject Classification. Primary 44A25; Secondary 42A18.

Key words and phrases. Hilbert transform, weighted norm inequalities, $A_{p}$ weights, functions with vanishing moments. 
complex variable techniques, which are not available for $p=2$. In this paper a real variable proof is given for $1<p<\infty$.

This result and the corollary in the last section of this paper show that weights of this form are the only ones satisfying the doubling condition, for which multiplier theorems can be proved for the usual class of multipliers and indicate that the weight functions in [8-10] are the natural weights to use.

We will use the following notation. For a nonnegative function $v(x)$ the set of functions $f$ which satisfy $\int|f(x)|^{p} v(x) d x<\infty$, will be denoted by $L_{v}^{p}$. This is a normed space with the norm

$$
\|f\|_{p, v}=\left(\int|f(x)|^{p} v(x) d x\right)^{1 / p} .
$$

By $L_{\text {loc }}^{1}$ we mean the set of locally integrable functions. The letters $k, m$ and $N$ will denote nonnegative integers and $C$ will always denote a positive constant, not necessarily the same at each occurrence.

2. The theorem to be proved is the following.

THEOREM (2.1). Given $p, 1<p<\infty$, and a nonnegative integer $N$, then a nonnegative, measurable function $w$ satisfies (1.1) for all $f \in L_{(1+|x|)^{N}}^{1}$ with $\int x^{k} f(x) d x=0$, $0 \leqslant k \leqslant N$, if and only if

$$
w(x)=\mid a_{0}+a_{1} x+\cdots+a_{N+1} x^{N+1} p U(x)
$$

with $a_{i}$ complex numbers and $U \in A_{p}$.

As already mentioned, the sufficiency proof is very easy. It only uses the fact that for the above $f$ 's $q \tilde{f}=(q f)^{\tilde{y}}$ for any polynomial $q$ of degree at most $N+1$. To see that this equality holds rewrite it as

$$
\int f(t) \frac{q(x)-q(t)}{x-t} d t=0 .
$$

This holds because $(q(x)-q(t)) /(x-t)$ is a polynomial in $t$ of degree less than or equal to $N$, so that the integral vanishes for each $x$. With this identity we get

$$
\int|\tilde{f}|^{p}|q|^{p} U=\int|q \tilde{f}|^{p} U=\left.\int\left|(q f)^{p} U \leqslant C \int\right| q f\right|^{p} U
$$

since $U \in A_{p}$. This proves the sufficiency.

The necessity part requires considerably more and will be done by considering three cases. We prove a slightly stronger version assuming (1.1) to hold only for bounded $f$ with compact support and $\int x^{k} f(x) d x=0,0 \leqslant k \leqslant N$.

To simplify notation let $H(N)$ denote the set of all nonnegative locally integrable functions $w$ which satisfy (1.1) for bounded $f$ with compact support and with the first $N+1$ moments zero for $N \geqslant 0$, and let $H(-1)$ stand for the set of all nonnegative locally integrable functions $w$ which satisfy (1.1) for bounded $f$ with compact support. After only slight modifications the proof in [6] shows that $H(-1)=A_{p}$. 
What we have to show now is that $w \in H(N)$ implies the existence of a polynomial $q$ of degree at most $N+1$ such that $w|q|^{-p} \in A_{p}$. Taking one step at a time it suffices to show that $w \in H(N)$ implies $w(x)|a x+b|^{-p} \in H(N-1)$ for some complex numbers $a$ and $b$.

To do this we consider the three cases:

(I) $w^{-1 /(p-1)}$ not locally integrable at some $c \in \mathbf{R}$,

(II) $w^{-1 /(p-1)}$ locally integrable, but $w^{-1 /(p-1)} \notin L^{1}$, (III) $w^{-1 /(p-1)} \in L^{1}$.

Presupposing the existence of the above $q$, (I)-(III) correspond to the cases:

$q$ has at least one real zero (at $c \in \mathbf{R}$ ),

$q$ has no zero, i.e. is a constant,

$q$ has only complex zeros and has at least one.

We will show that

(I) implies $w(x)|x-c|^{-p} \in H(N-1)$,

(II) implies $w \in H(N-1)$, and

(III) implies $w(x)|x-i|^{-p} \in H(N-1)$.

(For (III) we actually show $w(x)(1+|x|)^{-p} \in H(N-1)$, but this is obviously equivalent to $w(x)|x-i|^{-p} \in H(N-1)$.)

Before we come to the three cases, we show that if $w$ satisfies (1.1), then it has to be either locally integrable or $w=\infty$ a.e.

So let $w$ be finite on a set of positive measure. Then there exists a positive number $B$ and a bounded set $E$ with positive measure such that $w$ is less than $B$ on $E$. Now we state two lemmas whose proofs are virtually the same as that of Lemma (2.6) in [3].

Lemma (2.2). Given a measurable set $E \subset \mathbf{R}$ with finite positive measure and $N$ bounded measurable functions $\varphi_{k}$ on $E$ which are linearly independent over $E$, then for any sequence of complex numbers $\lambda_{k}, k=1, \ldots, N$, there exists a bounded measurable function $\alpha$ such that

$$
\int_{E} \alpha(x) \varphi_{k}(x) d x=\lambda_{k}, \quad k=1, \ldots, N .
$$

LEMMA (2.3). Given a finite interval I and $N$ functions $\varphi_{k}, C^{\infty}$ in a neighborhood of I and linearly independent over $I$, then for any complex numbers $\lambda_{k}$, there exists a $C^{\infty}$ function $\alpha$ with support in I with

$$
\int \alpha(x) \varphi_{k}(x) d x=\lambda_{k}, \quad k=1, \ldots, N .
$$

In the sequel we will denote the set of $C^{\infty}$ functions with support in a set $F$ by $C^{\infty}(F)$.

Lemma (2.2) guarantees the existence of a function $\alpha \in L^{\infty}(E)$ with

$$
\int_{E} x^{k} \alpha(x) d x= \begin{cases}0, & 0 \leqslant k \leqslant N \\ 1, & k=N+1\end{cases}
$$


But for such $\alpha|\tilde{\alpha}(x)| \sim|x|^{-N-2}$ as $|x| \rightarrow \infty$ (this will be shown soon), say $|\tilde{\alpha}(x)| \geqslant$ $\left|2 x^{N+2}\right|^{-1}$ for $|x| \geqslant M$, with $M$ so large that also $E \subset[-M, M]$. We have then

$$
\int_{|x|>M} w(x)|x|^{-(N+2) p} d x \leqslant C \int_{|x|>M}|\tilde{\alpha}(x)|^{p} w(x) d x \leqslant C \int|\alpha|^{p} w .
$$

Since the last term is bounded by $C\|\alpha\|_{\infty}^{p} B|E|$, this shows that $w$ is locally integrable in $\{|x| \geqslant M\}$.

Now let $\beta(x)=\alpha(x-2 M)$. Then $\beta$ is supported in $\{|x| \geqslant M\}$,

$$
\int x^{k} \beta(x) d x=0, \quad 0 \leqslant k \leqslant N,
$$

and

$$
|\tilde{\beta}(x)|=|\tilde{\alpha}(x-2 M)| \geqslant\left(2|x-2 M|^{N+2}\right)^{-1} \text { for }|x-2 M| \geqslant M .
$$

Thus

$$
\begin{aligned}
\int_{|x|<M} w(x) \mid x & -\left.2 M\right|^{-p(N+2)} d x \\
& \leqslant \int_{|x-2 M|>M} w(x)|x-2 M|^{-p(N+2)} d x \leqslant C \int|\tilde{\beta}|^{p} w
\end{aligned}
$$

Since the last term is bounded by a $C \int|\beta|^{p} w$, which is finite, because supp $\beta$ is contained in $\{|x| \geqslant M\}$, this proves the claim modulo the following.

OBSERVATION (2.4). For $\alpha \in L^{1}$ with compact support and

$$
\int x^{k} \alpha(x) d x= \begin{cases}0, & 0 \leqslant k \leqslant N \\ 1, & k=N+1,\end{cases}
$$

we have $|\tilde{\alpha}(x)| \sim|x|^{-N-2}$ as $|x| \rightarrow \infty$, or in other words

$$
\lim _{|x| \rightarrow \infty}|x|^{N+2}|\tilde{\alpha}(x)|=1 \text {. }
$$

Since for such $\alpha, x^{N+1} \tilde{\alpha}(x)=\left(t^{N+1} \alpha\right) \tilde{)}(x)$, we have to show that

$$
\left|x\left(t^{N+1} \alpha\right) \tilde{)}(x)\right| \rightarrow 1 \quad \text { as }|x| \rightarrow \infty,
$$

or that for any integrable $g$ with compact support and $\int g=1$

$$
x \tilde{g}(x) \rightarrow 1 \text { as }|x| \rightarrow \infty .
$$

This is obvious from the compact support of $g$.

We now proceed by treating the three cases separately.

Case I. We can assume that $c=0$ and that, for all positive $\sigma, \int_{0}^{\sigma} w^{-1 /(p-1)}=\infty$. Then we have to show that $w(x)|x|^{-p} \in H(N-1)$. The main part of the proof lies in the following

LEMMA (2.5). If $w \in H(N)$ and, for all positive $\sigma, \int_{0}^{\sigma} w^{-1 /(p-1)}=\infty$, then

$$
\int_{0}^{1} \frac{w(x)}{|x|^{p}} d x<\infty .
$$


Proof. We will need functions $\alpha_{i} \in C^{\infty}[2,3], i=0, \ldots, N$, with $\int x^{k} \alpha_{i}(x) d x=\delta_{i k}$, $0 \leqslant i, k \leqslant N$, and also $\int \alpha_{0}(x) d x / x=-\tilde{\alpha}_{0}(0)=1$. This existence is guaranteed by Lemma (2.3). Since $\tilde{\alpha}_{0}$ is continuous (its Fourier transform is in $L^{1}$ ), and $\tilde{\alpha}_{0}(0)<0$ we have that $\tilde{\alpha}_{0}$ is negative on $[-\tau, \tau]$ for some positive $\tau$. Since $w \in L_{\text {loc }}^{1}$ it is sufficient to show that

$$
\int_{0}^{\tau} \frac{w(x)}{|x|^{p}} d x<\infty
$$

For any positive number $\delta$ we will use the notation

$$
w_{\delta}(x)= \begin{cases}w(x), & w(x) \geqslant \delta \\ \delta, & w(x) \leqslant \delta .\end{cases}
$$

Now for any positive $\varepsilon$ by assumption we can find $0<\delta<\varepsilon$ such that $\int_{\delta}^{\varepsilon} w_{\delta}(x)^{-1 /(p-1)} d x=1$. Then we let

$$
f=w_{\delta}^{-1 /(p-1)} \chi[\delta, \varepsilon]-\sum_{i=0}^{N} \alpha_{i} \int_{\delta}^{\varepsilon} x^{i} w_{\delta}(x)^{-1 /(p-1)} d x .
$$

This $f$ satisfies $\int x^{k} f(x) d x=0,0 \leqslant k \leqslant N$, so that

$$
\int|\tilde{f}|^{p} w \leqslant C \int|f|^{p} w
$$

The right side is bounded by

$$
C \int_{\delta}^{\varepsilon} w_{\delta}^{-1 /(p-1)}+C \sum_{i=0}^{N} \int_{2}^{3}\left|\alpha_{i}\right| p w\left|\int_{\delta}^{\varepsilon} x^{i} w_{\delta}(x)^{-1 /(p-1)} d x\right|^{p},
$$

which is less than some $C_{0}$ independent of $\varepsilon$, if $\varepsilon<1$, since

$$
\int_{\delta}^{\varepsilon} x^{i} w_{\delta}(x)^{-1 /(p-1)} d x \leqslant \int_{\delta}^{\varepsilon} w_{\delta}(x)^{-1 /(p-1)} d x=1 .
$$

To estimate the left-hand side of (2.6) we first note that if $\varepsilon<\tau$ and if $x \in[\varepsilon, \tau]$, a lower bound for $|\tilde{f}(x)|$ is given by the difference of

$$
\left|\int_{\delta}^{\varepsilon} \frac{w_{\delta}(t)^{-1 /(p-1)}}{x-t} d t-\tilde{\alpha}_{0}(x) \int_{\delta}^{\varepsilon} w_{\delta}^{-1 /(p-1)}\right|
$$

and

$$
\sum_{i=1}^{N}\left|\tilde{\alpha}_{i}(x)\right| \int_{\delta}^{\varepsilon} x^{i} w_{\delta}(x)^{-1 /(p-1)} d x
$$

Since $\tilde{\alpha}_{0}$ is negative in $[\varepsilon, \tau],(2.7)$ is greater than

$$
\int_{\delta}^{\varepsilon} \frac{w_{\delta}(t)^{-1 /(p-1)}}{x-t} d t
$$

For (2.8) we have the upper bound

$$
C \sum_{i=1}^{N} \varepsilon^{i} \int_{\delta}^{\varepsilon} w_{\delta}^{-1 /(p-1)} .
$$


If $0<\varepsilon<\frac{1}{2}$ the difference of (2.9) and (2.10) is bounded below by $\frac{1}{x}-C \cdot 2 \varepsilon$. If $\varepsilon<\frac{1}{4 C}$ this is greater than $\frac{1}{x}-\frac{1}{2}$ which is greater than $\frac{1}{2 x}$, since $0<x<1$. So for small. $\varepsilon$ we have

$$
\int_{\varepsilon}^{\tau} \frac{w(x)}{|x|^{p}} d x \leqslant C \int|\tilde{f}|^{p} w \leqslant C \int|f|^{p} w \leqslant C_{0} .
$$

Letting $\varepsilon \rightarrow 0$ proves the lemma.

With the help of this lemma we can finish case I. An equivalent way of writing (1.1) is

$$
\int|\tilde{h}(x)|^{p} \frac{w(x)}{|x|^{p}} d x \leqslant C \int|h(x)|^{p} \frac{w(x)}{|x|^{p}} d x
$$

for $h(x) / x$ bounded, compact support and

$$
\int x^{k} \frac{h(x)}{x} d x=0, \quad k=0, \ldots, N .
$$

To see this just use $\tilde{h}(x) / x=\left(h(t) / t \tilde{)}(x)\right.$, since $\int h(x) / x d x=0$.

To show now that $w(x) /|x|^{p} \in H(N-1)$, let $g$ have compact support not containing the origin and satisfy

$$
\int x^{k} g(x) d x=0, \quad k=0, \ldots, N-1 .
$$

Let $g_{m}(x)=g(x)-m x \alpha_{0}(m x) \int g(x) / x d x$, where $\alpha_{0} \in C^{\infty}[0,1], \int x^{k} \alpha_{0}(x) d x=$ $\delta_{0, k}, k=0, \ldots, N$. Then $\int x^{k} g_{m}(x) / x d x=0, k=0, \ldots, N$, and thus by the above remark

$$
\int\left|\tilde{g}_{m}(x)\right|^{p} \frac{w(x)}{|x|^{p}} d x \leqslant C \int\left|g_{m}(x)\right|^{p} \frac{w(x)}{|x|^{p}} d x .
$$

Since $\left[m t \alpha_{0}(m t) \tilde{]}(x)=\left[t \alpha_{0} \tilde{]}(m x) \rightarrow 0\right.\right.$ for a.e. $x$ as $m \rightarrow \infty$ we have $\tilde{g}_{m} \rightarrow \tilde{g}$ a.e. and using Fatou's lemma and (2.12) we get

$$
\begin{aligned}
\int|\tilde{g}(x)|^{p} \frac{w(x)}{|x|^{p}} d x & \leqslant \liminf _{m \rightarrow \infty} \int\left|\tilde{g}_{m}(x)\right|^{p} \frac{w(x)}{|x|^{p}} d x \\
& \leqslant C \liminf _{m \rightarrow \infty} \int\left|g_{m}(x)\right|^{p} \frac{w(x)}{|x|^{p}} d x .
\end{aligned}
$$

This is bounded by

$$
C \int|g(x)|^{p} \frac{w(x)}{|x|^{p}} d x+C \liminf _{m \rightarrow \infty} \int_{0}^{1 / m}\left|m x \alpha_{0}(m x)\right|^{p} \frac{w(x)}{|x|^{p}} d x \cdot\left|\int \frac{g(x)}{x} d x\right|^{p} .
$$

But the last term is zero since $\left|m x \alpha_{0}(m x)\right|$ is bounded by $\left\|x \alpha_{0}(x)\right\|_{\infty}$ uniformly in $m$ and because of the lemma.

To complete Case I we have to remove the restriction that zero should not be contained in the support of $g$. So let $g \in L_{(1+|x|)^{N-1}}^{1}$ be arbitrary and satisfy (2.11). Let

$$
h_{m}=g \chi\left\{|x|>\frac{1}{m}\right\}+\sum_{i=0}^{N-1} \alpha_{i} \int_{-1 / m}^{1 / m} x^{i} g(x) d x
$$


where $\alpha_{i} \in C^{\infty}[1,2], \int x^{k} \alpha_{i}(x) d x=\delta_{i k}, 0 \leqslant i, k \leqslant N-1$. Then $h_{m}$ satisfies (2.11) and zero is not contained in the support of $h_{m}$, so that

$$
\int\left|\tilde{h}_{m}(x)\right|^{p} \frac{w(x)}{|x|^{p}} d x \leqslant C \int\left|h_{m}(x)\right|^{p} \frac{w(x)}{|x|^{p}} d x .
$$

Since $\tilde{h}_{m}-\tilde{g}$ equals

$$
\left(-g \chi\left\{|x|<\frac{1}{m}\right\}\right) \sim+\sum_{i=0}^{N-1} \tilde{\alpha}_{i} \int_{-1 / m}^{1 / m} x^{i} g(x) d x
$$

which goes to zero a.e. as $m \rightarrow \infty$, we have using Fatou's lemma again and (2.13)

$$
\begin{aligned}
\int|\tilde{g}(x)|^{p} \frac{w(x)}{|x|^{p}} d x & \leqslant \liminf _{m \rightarrow \infty} \int\left|\tilde{h}_{m}(x)\right|^{p} \frac{w(x)}{|x|^{p}} d x \\
& \leqslant C \liminf _{m \rightarrow \infty} \int\left|h_{m}(x)\right|^{p} \frac{w(x)}{|x|^{p}} d x .
\end{aligned}
$$

This is bounded by

$$
C \int|g(x)|^{p} \frac{w(x)}{|x|^{p}} d x+C \sum_{i=0}^{N-1} \int\left|\alpha_{i}\right|^{p} w \cdot \liminf _{m \rightarrow \infty}\left|\int_{-1 / m}^{1 / m} x^{i} g(x) d x\right|^{p} .
$$

This proves Case I, since the last term is zero.

Case II. We consider now the case that $w^{-1 /(p-1)}$ is locally integrable but that, say, $\int_{0}^{\infty} w^{-1 /(p-1)}=\infty$. As in the preceding case there is a lemma, which contains the heart of the proof.

LEMMA (2.14). If $w \in H(N), w^{-1 /(p-1)}$ locally integrable and $\int_{0}^{\infty} w^{-1 /(p-1)}=\infty$, then $\int_{-\infty}^{0} w(x) /\left(1+|x|^{p(N+1)}\right) d x<\infty$.

Proof. For positive $h$ we will again use the notation

$$
w_{1 / h}(x)= \begin{cases}w(x), & w(x)>1 / h \\ 1 / h, & w(x) \leqslant 1 / h\end{cases}
$$

Then given any $M$ greater than 1 , by the assumption we can find a positive $h$ such that

$$
\int_{M}^{M+h} x^{N} w_{1 / h}(x)^{-1 /(p-1)} d x=1
$$

Let then

$$
f=w_{1 / h}^{-1 /(p-1)} \chi[M, M+h]-\sum_{i=0}^{N} \alpha_{i} \int_{M}^{M+h} t^{i} w_{1 / h}(t)^{-1 /(p-1)} d t
$$

where $\alpha_{i} \in C^{\infty}[0,1], \int x^{k} \alpha_{i}(x) d x=\delta_{i k}, 0 \leqslant i, k \leqslant N$. Then $f$ satisfies $\int x^{k} f(x) d x=$ $0, k=0, \ldots, N$, and thus

$$
\int|\tilde{f}(x)|^{p} w(x) d x \leqslant C \int|f(x)|^{p} w(x) d x
$$


The last term is bounded above by

$$
C \int_{M}^{M+h} w_{1 / h}^{-1 /(p-1)}+C \sum_{i=0}^{N} \int_{0}^{1}\left|\alpha_{i}\right|^{p} w\left|\int_{M}^{M+h} t^{i} w_{1 / h}(t)^{-1 /(p-1)} d t\right|^{p}
$$

which is less than some constant $C_{0}$ independent of $M$, since

$$
\int_{M}^{M+h} t^{i} w_{1 / h}(t)^{-1 /(p-1)} d t \leqslant \int_{M}^{M+h} t^{N} w_{1 / h}(t)^{-1 /(p-1)} d t=1, \quad 0 \leqslant i \leqslant N .
$$

To estimate the left-hand side of (2.15) we note first that $|\tilde{f}(x)|$ is greater than or equal to the difference of $\left|\tilde{\alpha}_{N}(x)\right|$ and

$$
\sum_{i=0}^{N-1}\left|\tilde{\alpha}_{i}(x)\right| \int_{M}^{M+h} t^{i} w_{1 / h}(t)^{-1 /(p-1)} d t+\left|\int_{M}^{M+h} w_{1 / h}(t)^{-1 /(p-1)} \frac{d t}{x-t}\right|
$$

By observation (2.4) $\left|\tilde{\alpha}_{N}(x)\right|$ is bounded below by $\left|2 x^{N+1}\right|^{-1}$ for large $x$, say $|x|>r, r$ depending only on $\alpha_{N}$.

The first term of (2.16) is bounded above by

$$
\sum_{i=0}^{N-1}\left\|\tilde{\alpha}_{i}\right\|_{\infty} \frac{1}{M^{N-i}} \int_{M}^{M+h} t^{N} w_{1 / h}(t)^{-1 /(p-1)} d t
$$

which is less than $C / M$ for some $C$, since $M>1$.

If we only consider negative $x$, then the second term of (2.16) is less than

$$
\frac{1}{M} \int_{M}^{M+h} w_{1 / h}^{-1 /(p-1)} \leqslant \frac{1}{M} \text {. }
$$

So altogether for $x<-r$ :

$$
|\tilde{f}(x)| \geqslant \frac{1}{2|x|^{N+1}}-\frac{C}{M} .
$$

This is greater than $\left|4 x^{N+1}\right|^{-1}$ for $|x|<M^{\prime}=(M / 4 C)^{1 /(N+1)}$. This gives $\int_{-M^{\prime}}^{-r} w(x) /|x|^{p(N+1)} d x \leqslant C \int \mid \tilde{f}^{p} w \leqslant C_{0}$. Letting $M \rightarrow \infty$, hence $M^{\prime} \rightarrow \infty$ and observing that $w \in L_{\text {loc }}^{1}$ proves the lemma.

Now we can finish Case II. We want to show that $w \in H(N-1)$. So let $g$ be a bounded function with compact support and let it satisfy

$$
\int x^{k} g(x) d x=0, \quad k=0, \ldots, N-1
$$

Let

$$
g_{m}(x)=g(x)-\frac{\alpha_{N}(x / m)}{m^{N+1}} \int t^{N} g(t) d t,
$$

where $\alpha_{N} \in C^{\infty}[-2,-1]$, such that $\int x^{k} \alpha_{N}(x) d x=\delta_{k N}, k=0, \ldots, N$. Then $g_{m}$ satisfies $\int x^{k} g_{m}(x) d x=0, k=0, \ldots, N$ so that

$$
\int\left|\tilde{g}_{m}\right|^{p} w \leqslant C \int\left|g_{m}\right|^{p} w
$$

Since $\tilde{g}(x)-\tilde{g}_{m}(x)=\left(\tilde{\alpha}_{N}(x / m) / m^{N+1}\right) \int t^{N} g(t) d t$ tends to zero for all $x$ as $m$ 
goes to infinity we get using Fatou's lemma and (2.17)

$$
\int|\tilde{g}|^{p} w \leqslant \liminf _{m \rightarrow \infty} \int\left|\tilde{g}_{m}\right|^{p} w \leqslant C \liminf _{m \rightarrow \infty} \int\left|g_{m}\right|^{p} w
$$

This is bounded by

$$
C \int|g|^{p} w+C \liminf _{m \rightarrow \infty} \int_{-2 m}^{-m} \frac{\left|\alpha_{N}(x / m)\right|^{p}}{m^{p(N+1)}} w(x) d x\left|\int t^{N} g(t) d t\right|^{p} .
$$

Since $\alpha_{N}$ is bounded and

$$
\lim _{m \rightarrow \infty} \int_{-2 m}^{-m} \frac{w(x)}{|x|^{p(N+1)}} d x=0
$$

by the lemma, the proof of Case II is complete.

Case III. The case where $w^{-1 /(p-1)} \in L^{1}$ is the easiest of the three and can be treated by the methods of Cases I and II. We want to show that $w(x) /\left(1+|x|^{p}\right)^{-1}$ $\in H(N-1)$, so let $g$ be a bounded function with compact support and

$$
\int x^{k} g(x) d x=0, \quad 0 \leqslant k \leqslant N-1 .
$$

Let $\alpha_{i} \in C^{\infty}[1,2], \int x^{k} \alpha_{i}(x) d x=\delta_{i k}, 0 \leqslant i, k \leqslant N+1$. Write $g=g_{0}+g_{1}$, where

$$
g_{0}=g \chi[-2,2]-\sum_{i=0}^{N-1} \alpha_{i} \int_{-2}^{2} t^{i} g(t) d t
$$

and

$$
g_{1}=g \chi\{|x|>2\}+\sum_{i=0}^{N-1} \alpha_{i} \int_{-2}^{2} t^{i} g(t) d t
$$

Then both $g_{0}$ and $g_{1}$ satisfy (2.18).

Now we estimate

$$
\int\left|\tilde{g}_{0}(x)\right|^{p} \frac{w(x)}{1+|x|^{p}} d x \text { and } \int\left|\tilde{g}_{1}(x)\right|^{p} \frac{w(x)}{1+|x|^{p}} d x
$$

separately. We will make use of the following observation. If $w \in H(N)$ then

$$
\int \frac{w(x)}{1+|x|^{p(N+2)}} d x<\infty .
$$

This follows if we take $f$ to be $\alpha_{N+1}$ in (1.1), because then $|\tilde{f}(x)| \sim|x|^{-N-2}$ at infinity.

Let first $f=g_{0}-\alpha_{N} \int t^{N} g_{0}(t) d t$, so that

$$
\int x^{k} f(x) d x=0, \quad 0 \leqslant k \leqslant N .
$$

Thus

$$
\int|\tilde{f}|^{p} w \leqslant C \int|f|^{p} w
$$


Now we can estimate

$$
\int\left|\tilde{g}_{0}(x)\right|^{p} \frac{w(x)}{1+|x|^{p}} d x
$$

by

$$
\int|\tilde{f}(x)|^{p} \frac{w(x)}{1+|x|^{p}} d x+\int\left|\tilde{\alpha}_{N}(x)\right|^{p} \frac{w(x)}{1+|x|^{p}} d x\left|\int t^{N} g_{0}(t) d t\right|^{p}
$$

Using the fact that $\left|\tilde{\alpha}_{N}(x)\right| \sim|x|^{-N-1}$ at infinity together with (2.19) and (2.21) we see that this is bounded above by

$$
C \int|f|^{p} w+C\left|\int t^{N} g_{0}(t) d t\right|^{p}
$$

The definition of $f$ gives the upper bound

$$
C \int\left|g_{0}\right|^{p} w+\left.C \int_{1}^{2}\left|\alpha_{N} p^{p} w\right| \int t^{N} g_{0}(t) d t\right|^{p}+C\left|\int t^{N} g_{0}(t) d t\right|^{p} .
$$

Since $g_{0}$ is supported in $[-2,2]$ this is less than

$$
C \int\left|g_{0}(x)\right|^{p} \frac{w(x)}{1+|x|^{p}} d x+C\left(\int\left|g_{0}\right|\right)^{p} .
$$

Hölder's inequality shows that the last term in (2.22) is bounded by

$$
C \int\left|g_{0}\right|^{p} w\left(\int w^{-1 /(p-1)}\right)^{p-1}
$$

which is less than

$$
C \int\left|g_{0}(x)\right|^{p} \frac{w(x)}{1+|x|^{p}} d x
$$

since $w^{-1 /(p-1)} \in L^{1}$. Using the definition of $g_{0}$ shows that this is bounded by the sum of

$$
C \int|g(x)|^{p} \frac{w(x)}{1+|x|^{p}} d x
$$

and

$$
C \sum_{i=0}^{N-1} \int_{1}^{2}\left|\alpha_{i}(x)\right|^{p} \frac{w(x)}{1+|x|^{p}} d x\left|\int_{-2}^{2} t^{i} g(t) d t\right|^{p} .
$$

To complete the $g_{0}$ part we estimate (2.24) by (2.23). First we get the bound

$$
C\left(\int \frac{g(x)}{1+|x|} d x\right)^{p}
$$

Using Hölder's inequality this is less than

$$
C \int|g(x)|^{p} \frac{w(x)}{1+|x|^{p}} d x\left(\int w^{-1 /(p-1)}\right)^{p-1} \leqslant C \int|g(x)|^{p} \frac{w(x)}{1+|x|^{p}} d x .
$$


Now we turn to the $g_{1}$ part. To estimate

$$
\int\left|\tilde{g}_{1}(x)\right|^{p} \frac{w(x)}{1+|x|^{p}} d x
$$

we let

$$
f(x)=\frac{g_{1}(x)}{x}-\alpha_{0}(x) \int \frac{g_{1}(t)}{t} d t .
$$

Then $\int x^{k} f(x) d x=0,0 \leqslant k \leqslant N$, hence

$$
\int|\tilde{f}|^{p} w \leqslant C \int|f|^{p} w
$$

Also

$$
x \tilde{f}(x)=(t f) \tilde{(}(x)=\tilde{g}_{1}(x)-\left(t \alpha_{0}\right) \tilde{(}(x) \int \frac{g_{1}(t)}{t} d t
$$

First we have

$$
\int\left|\tilde{g}_{1}(x)\right| p \frac{w(x)}{1+|x|^{p}} d x \leqslant C \int\left|\frac{x}{1+|x|} \frac{\tilde{g}_{1}(x)}{x}\right|^{p} w(x) d x
$$

Using (2.26) gives the upper bound

$$
C \int|\tilde{f}|^{p} w+C \int\left|\frac{\left(t \alpha_{0}\right) \tilde{(}(x)}{1+|x|}\right|^{p} w(x) d x\left|\int \frac{g_{1}(t)}{t} d t\right|^{p} .
$$

Now use (2.25), the fact that $\mid\left(\left.t \alpha_{0} \tilde{)}(x)|\sim| x\right|^{-N-1}\right.$ at infinity together with (2.19) and that $g_{1}$ is supported in $\{|x|>1\}$ to see that this is less than

$$
C \int|f|^{p} w+C\left(\int \frac{\left|g_{1}(t)\right|}{1+|t|} d t\right)^{p}
$$

The definition of $f$ and the fact that $\alpha_{0} \in C^{\infty}[1,2]$ give the bound

$$
C \int\left|g_{1}(x)\right|^{p} \frac{w(x)}{1+|x|^{p}} d x+C\left(\int \frac{\left|g_{1}(x)\right|}{1+|x|} d x\right)^{p} \text {. }
$$

Using Hölder's inequality and the fact that $w^{-1 /(p-1)} \in L^{1}$ shows that (2.27) is less than

$$
C \int\left|g_{1}(x)\right|^{p} \frac{w(x)}{1+|x|^{p}} d x
$$

This is less than

$$
C \int|g(x)|^{p} \frac{w(x)}{1+|x|^{p}} d x+C \int\left|g_{0}(x)\right|^{p} \frac{w(x)}{1+|x|^{p}} d x .
$$

But this is bounded by

$$
C \int|g(x)|^{p} \frac{w(x)}{1+|x|^{p}} d x
$$

using the above estimate for $g_{0}$. This completes Case III and therefore the theorem is proved. 
3. In [8-10] the authors consider weighted $L^{p}$ and $H^{p}$ spaces. They provide sufficient conditions on multipliers on these spaces to be bounded operators and show relationships between the weighted $L^{p}$ and $H^{p}$ spaces, respectively.

Their weights have the form $w(x)=|q(x)|^{p} U(x)$, where $q$ is a polynomial and $U \in A_{p}$. They prove norm inequalities with these weights for $f \in S_{0,0}$, the space of all rapidly decreasing $C^{\infty}$ functions, whose Fourier transform has compact support not containing the origin.

Since the multiplier of the Hilbert transform is a member of all the classes of multipliers considered in [8,9], and because of a little argument given in [10] the following corollary shows that under a weak assumption on $w$ the weight is necessarily of the above form.

Corollary (3.1). If

$$
\int|\tilde{f}|^{p} w \leqslant C \int|f|^{p} w
$$

for some $p, 1<p<\infty$, and for all $f \in S_{0,0}$, and if for some $N \geqslant-1$

$$
\int \frac{w(x)}{1+|x|^{p(N+2)}} d x<\infty
$$

then $w(x)=|q(x)|^{p} U(x)$, where $q$ is a polynomial of degree at most $N+1$ and $U \in A_{p}$.

Proof. In view of the theorem it suffices to show that $w$ satisfies (3.2) for bounded functions $f$ with compact support for which $\int x^{k} f(x) d x=0,0 \leqslant k \leqslant N$.

First we show that (3.3) implies

$$
\lim _{n \rightarrow \infty} n^{-p(N+2)} \int_{-n}^{n} w(x) d x=0 .
$$

For a given $\varepsilon>0$ choose $r$ such that

$$
\int_{|x|>r} \frac{w(x)}{|x|^{p(N+2)}} d x<\varepsilon
$$

Now let $n>r$ be so large that

$$
\int_{|x|<r} \frac{w(x)}{n^{p(N+2)}} d x<\varepsilon
$$

Then we have altogether

$$
\begin{aligned}
\int_{|x|<n} \frac{w(x)}{n^{p(N+2)}} d x & =\int_{|x|<r} \frac{w(x)}{n^{p(N+2)}} d x+\int_{r<|x|<n} \frac{w(x)}{n^{p(N+2)}} d x \\
& \leqslant \varepsilon+\int_{r<|x|<n} \frac{w(x)}{|x|^{p(N+2)}} d x \leqslant 2 \varepsilon .
\end{aligned}
$$

Thus we can apply Theorem (6.13) of [7] to get a sequence of functions $f_{n}$ in $S_{0,0}$ that converges to the above $f$ in $L^{2}$ and $L_{w}^{p}$. Since then $\tilde{f}_{n}$ converges to $\tilde{f}$ in $L^{2}$, we can assume that $\tilde{f}_{n}$ converges to $\tilde{f}$ a.e. Now we apply Fatou's lemma to get

$$
\int|\tilde{f}|^{p} w \leqslant \liminf _{n \rightarrow \infty} \int\left|\tilde{f}_{n}\right|^{p} w
$$


Since $f_{n} \in S_{0,0}$ this is bounded by

$$
C \liminf _{n \rightarrow \infty} \int\left|f_{n}\right|^{p} w=C \int|f|^{p} w
$$

This proves the corollary.

The following lemma shows that for locally integrable $w$ condition (3.3) is satisfied for instance if $w$ only satisfies the doubling condition, i.e. if for any interval $I$

$$
\int_{2 I} w(x) d x \leqslant D \int_{I} w(x) d x
$$

with $D$ independent of $I$. By $2 I$ we mean the interval which is twice as long as $I$ and has the same center.

LEMMA (3.5). If $w$ satisfies the doubling condition, then there exists $N>0$ such that

$$
\int \frac{w(x)}{1+|x|^{N}} d x<\infty
$$

Proof. From (3.4) it follows that for any $k$

$$
\int_{2^{k} I} w \leqslant D^{k} \int_{I} w
$$

Let $N>0$ be such that $D<2^{N}$. First

$$
\int \frac{w(x)}{1+|x|^{N}} d x \leqslant \int_{|x|<1} w(x) d x+\sum_{k=0}^{\infty} \int_{2^{k}<|x|<2^{k+1}} \frac{w(x)}{1+|x|^{N}} d x .
$$

Each integral under the sum sign can be estimated from above by

$$
2^{-k N} \int_{2^{k}<|x|<2^{k+1}} w(x) d x .
$$

Enlarging the range of integration and observing (3.6) gives the bound

$$
2^{-k N} D^{k} \int_{|x|<2} w(x) d x
$$

Since $D<2^{N}$ this shows that (3.7) is finite which completes the proof.

If no growth condition on $w$ is imposed in Corollary (3.1), there might not exist any nonzero $f \in S_{0,0}$ for which $\int|f|^{p} w$ is finite, so that (3.2) is trivially true. In that case nothing can be said about the form of $w$.

Take for example $w(x)=e^{a|x|}$ for some $a>0$ and assume that for some $f \in S_{0,0}$ $\int|f|^{p} w$ is finite. Then Hölder's inequality shows that

$$
\int|f(x)| e^{a|x| / 2 p} d x<\infty
$$

Thus the Fourier transform $\hat{f}$ of $f$ is analytic in the strip $\{\xi+i \eta|| \eta \mid<a / 2 p\}$. But since $\hat{f}$ vanishes in a neighborhood of the origin, $\hat{f}$ is identically zero.

What the best condition on $w$ is to ensure that $\int|f|^{p} w$ is finite for at least one nonzero $f \in S_{0,0}$ is unknown to the author. A good guess is probably

$$
\int \frac{\log w(x)}{1+x^{2}} d x<\infty
$$

See [2] for results in this direction. 


\section{REFERENCES}

1. R. Arocena, M. Cotlar and C. Sadosky, Weighted inequalities in $L^{2}$ and lifting properties, Adv. in Math. (to appear).

2. A. Beurling and P. Malliavin, On Fourier transforms of measures with compact support, Acta Math. 107 (1962), 291-309.

3. A. P. Calderon and A. Zygmund, Local properties of solutions of elliptic partial differential equations, Studia Math. 20 (1961), 171-225.

4. H. Helson and D. Sarason, Past and future, Math. Scand. 21 (1967), 5-16.

5. H. Helson and G. Szegö, A problem in prediction theory, Ann. Mat. Pura Appl. (4) 51 (1960), $107-138$.

6. R. Hunt, B. Muckenhoupt and R. Wheeden, Weighted norm inequalities for the conjugate function and Hilbert transform, Trans. Amer. Math. Soc. 176 (1973), 227-251.

7. B. Muckenhoupt, R. Wheeden and W. Young, $L^{2}$ multipliers with power weights, Adv. in Math. (to appear).

8. Weighted $L^{p}$ multipliers (to appear).

9. J.-O. Strömberg and A. Torchinsky, Weighted Hardy spaces (to appear).

10. J.-O. Strömberg and R. Wheeden, Relations between $H_{u}^{p}$ and $L_{u}^{p}$ with polynomial weights (to appear).

Department of Mathematics, Rutgers University, New Brunswick, New Jersey 08903

Current address: Department of Mathematics, University of Texas, Austin, Texas 78712 